\title{
On the Situated Semantics of Service Systems (Short paper)
}

\author{
Anshuman Saxena ${ }^{1,2}$ \\ ${ }^{1}$ LAMS, I\&C, EPFL Switzerland \\ ${ }^{2}$ TCS Innovation Labs, Bangalore India \\ anshuman.saxena@\{epfl.ch,tcs.com
}

\author{
Alain Wegmann \\ Systemic Modeling Laboratory (LAMS) \\ I\&C, EPF-Lausanne Switzerland \\ alain.wegmann@epfl.ch
}

\begin{abstract}
Service System refers to the group of entities that work together to implement a service. An important challenge for the service designer is to organize her conceptualization of the service in a way that helps her identify the functional components required to engineer the service. At a basic level of conceptualization, the functional relevance of an observed category can be historically inspired, empirically informed, rationally thought, or pragmatically focused. From a servicedesign perspective, the interest in a given phenomenon is limited to its functional organization, which requires taking an exclusively pragmatic view of the world. In this paper, we propose a role-based approach to modeling categories, which requires service-designers assign functional and non-functional roles to categories by making explicit their interpretation of the conceptual relevance of these categories. Staying aware of the design choices will help the service-designer develop an informed model of observed reality, leading to better alignment between the scope and the purpose of the design activity.
\end{abstract}

Keywords-critical reflection; cognitive grounding; situated role assignment; functional roles

\section{INTRODUCTION}

Systems is a set of interacting categories, which together exhibit some behavior for an observer, and that this behavior cannot be construed from any subset of these categories [1]. Further, categories are not observed in isolation. They are revealed as part of a conceptualization process, which invokes the image of some earlier experienced situation [2]. The imagistic character of conceptualization is inspired by multiple epistemic considerations, which may assign functional or non-functional roles to the observed categories [3]. From a service-design perspective, the interest in a given phenomenon is limited to the causal organization of the phenomenon. Service-design is an engineering endeavor to enrich some aspect of the real world through a man-made artifact [4]. As a result, a service-oriented systems model of the phenomenon of interest reifies service-systems by admitting only those categories, which have some functional relevance to the phenomenon of interest.

In this paper, we highlight the need for service-designers to critically reflect on the imagery that the phenomenon of interest invokes and reason why one sees the categories that she sees. It is important to note that this paper uses the concept of image to refer to the semantics of a situation and not any specific digital artifact. In that sense, the image as a whole provides the context for interpreting the relevance of the observed categories. We refer to the four prominent epistemic considerations, described in [3], and demonstrate how they aid in reasoning the conceptual relevance of the observed categories to ascertain if these categories have some functional relevance to the imagined situation.

\section{The IMAGistic NATURE OF CONCEPTUALIZATION}

Cognitively, conceptualization is situated [2]. Situatedness refers to the imagery that conceptualization invokes. It is the reenactment of a combination of prior experiences that together simulate a perceptual experience in the form of a situation - experienced or imaginary. The imagistic nature of conceptualization is enabled through different epistemic considerations. The four prominent epistemological theories that are considered relevant from an information science perspective include historicism, empiricism, rationalism and pragmatism. It is the contribution of these different epistemological views in activating an imagined situation that lends semantics its encyclopedic nature [5].

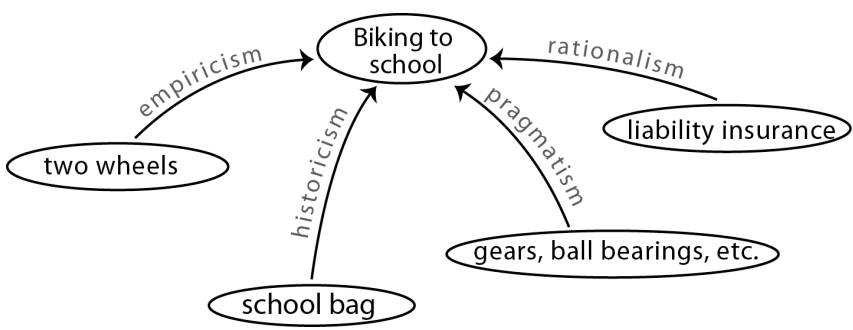

Figure 1: Epistemological grounding of an example conceptualization

For example, consider the case of a bike manufacturer conducting an inquiry on the use of bike to go to school. One popular image of a person biking to school is where she carries a bag. The inclusion of bag in the 'bike to school' conceptualization may be grounded in historicism, as historically we, as students, have been exposed to the act of carrying reading, writing instruments and lunch box to school. From a bike manufacturer's perspective, the role of 'bag-carrier' undertaken by the person biking to school is not, by default, relevant. This, initially historicism inspired, role gains significance for the inquiry only when it is shown to have some functional relevance to the 'bike to school' phenomenon; say, increased tiredness of the biker due to the weight of the bag on the shoulder, reduced maneuverability in traffic, ergonomic considerations, etc. Fig. 1 highlights the 
different epistemic groundings that may contribute to the popular imagery of an individual biking to school. In the following we refer to [3] in providing a detailed account of these epistemological groundings.

\section{A. Empricism}

Empiricism is that epistemic influence on conceptualization, where judgment is based solely on observations. For example, the popular image of bike as having two wheels is due to the fact that our exposure to bike designs with two wheels is considerably higher amongst our overall experience of the bike as a concept. Such number-ofexposure based reinforcement of a concept is referred to as grounded in empiricist consideration.

\section{B. Rationalism}

Rationalism is that epistemic influence on conceptualization, which is grounded in the tradition of logic. Rationally inspired conceptualization exposes the mental model of the modeler who builds the logical argument to deduce a specific inference. For example, the inclusion of liability insurance as an element of the popular image of bike reveals the authors' mental model of biking, say, in a country like Switzerland where most risks are appropriated.

\section{Historicism}

Historicism is that epistemic influence on conceptualization, which is based on social contexts and historical developments. It relies on culturally grounded experience that is accumulated overtime. For example, the inclusion of bag in the 'biking to school' conceptualization may be grounded in historicism, as historically we have been exposed to the act of carrying reading, writing instruments and lunch box to school. This may not necessarily hold today as adoption of IT based teaching methods have made stationary redundant and home-packed lunch has made way for cafeteria food.

\section{Pragmatism}

Pragmatism is that epistemic consideration which is driven by the purpose of the conceptualization. It promotes a functional view of reality where categories are acknowledged by virtue of their relevance to explaining the causal organization of the phenomenon of interest. For example, the inclusion of ball bearings and gear as part of popular imagery of the bike as a means of transport, exhibits the bike manufacturers' purpose of conducting the inquiry to come up with new bike designs.

Thus, the categories revealed in a conceptualization may not always undertake a functional role. They may also undertake non-functional roles that contribute to the imagery at the structural level [6].

\section{CRITICAL REFLECTION}

The categories observed in the real world can have several different cognitive groundings. A category can be perceptually relevant to the observer due to its role in formoriented completion of the imagery. Form-oriented synthesis of reality is widely studied as part of the Gestalt movement in psychology [6]. Gestalt refers to the unity of human experience. The claim of Gestalt Theory is that humans experience wholes not parts, and the whole is more than the sum of its parts. For example, when looking at a complex arrangement of individual elements, humans tend to first look for a single, recognizable pattern. There is, therefore, a constant effort to categorize observed reality at a level of granularity that provides a cognitive closure of sorts and thus lead to unity of experience. For example, Figure-ground organization is a type of perceptual grouping, which is vital for recognizing objects through vision. Elements are perceived as either figures -distinct elements of focus, or ground - the background or landscape on which the figures rests. Other prominent patterns of Gestalt completion include Base-profile for uniformity-oriented completion and Cognitive reference for proximity-oriented completion. Gestalt formation is a functional activity as far as the synthesis of experience is concerned but does not contribute functionally to the cause-and-effect relationships that constitute the semantics of the phenomenon.

To ascertain if the observed category has an exclusively Gestalt role or is also influencing the observed behavior through some cause-and-effect relationship, we recommend the service-designer reflects on the nature of the conceptual relevance that the observed categories may have for her. The questions that she can pose to herself are:

Why do I see, what I see? How is this relevant to my inquiry?

- Is it because I am used to seeing this aspect in this situation, but don't know why?

- Is it because I am used to seeing this aspect in this situation, and that is because of my social or cultural exposure to a specific kind of world, which includes beliefs/upbringing/atmosphere?

- Is it because that is how I think it should be? Whenever you see this one thing, there has to be this other thing, whether explicitly mentioned or not - its logical.

- Is it because this has some relevance to the purpose of my inquiry? And the relevance can be explained in terms of a cause-and-effect relation.

These questions help the service-designer identify the epistemic groundings of the observed categories by reflecting if the conceptualization of the category is empirically informed, historically inspired, rationally thought or pragmatically focused. As is evident from these questions, it is only the pragmatically grounded category that contributes to the causal understanding of the phenomenon of interest. Fig. 2, provides a process-oriented overview of the approach suggested to identify the cognitive grounding of categories and thereby assign functional or non-functional roles.

\section{An Illustration of a Situated Service System}

In this section we develop a service-design scenario, which is representative of the real world problems that a 


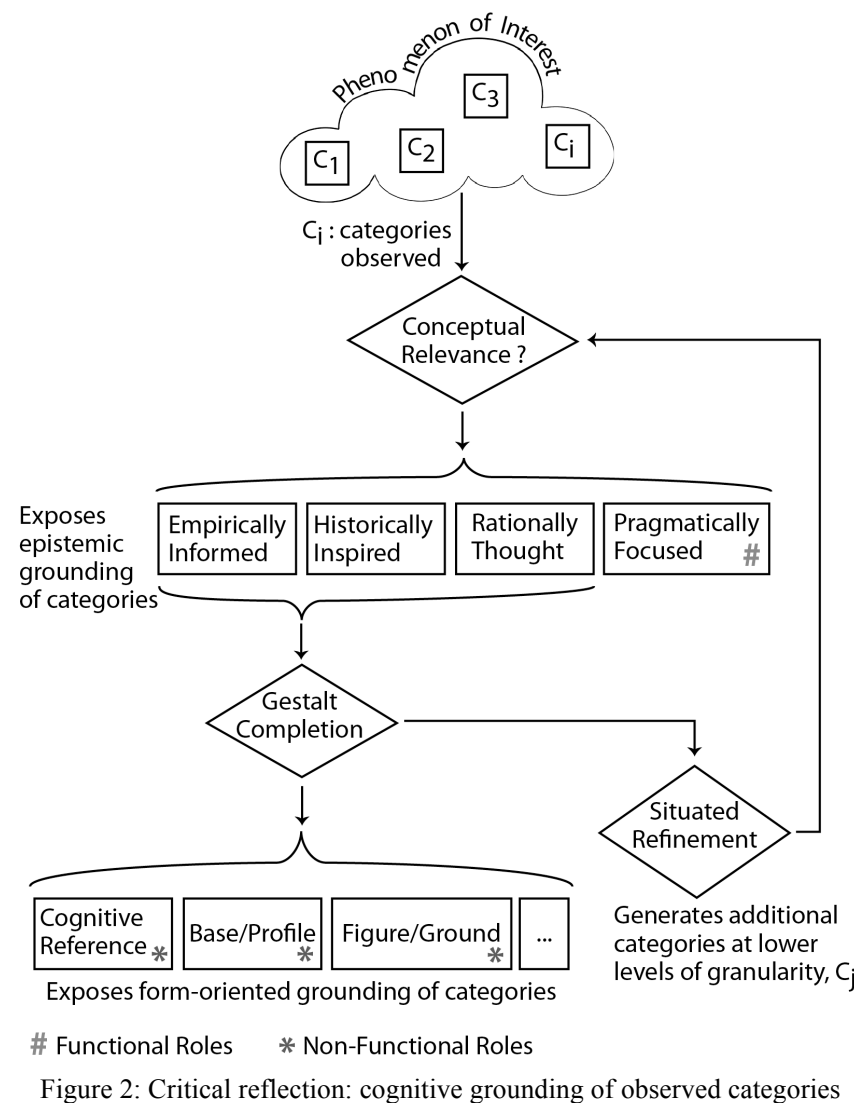

modeler has to address while specifying a service. The objective is to illustrate the process of critical reflection and demonstrate how it helps in identifying service-systems.

\section{A. Case Description}

The purpose of inquiry we undertake is to study the impact of the use of foldable-bike on the adoption of mixedmode of transport. Mixed-mode of transport refers to mobility solutions that involve the use of more than one mode of transport. For the purpose of this study we restrict ourselves to the combination of bike and train. Foldable-bike refers to those bike designs, which have a collapsible frame, whereby when not in use their form-factor can be reduced to a shape and size comparable with a conventional luggagebag allowed on board most public transports. The idea is to model the behavior that a foldable-bike manufacturer intends to realize for the people using these bikes. For the purpose of exposition, we limit the scope of our model to include only those users of foldable-bike who avail mixed-mode of transport for their commute to the workplace. More concretely, we assume the Focal-element here to be the user in her role as an office-worker. It is important to note that there is no loss of generality here due to this simplifying assumption. Our focus here is on identifying the servicesystem, which intends to realize a behavior that creates value for the user, possibly in several different ways - one of which we highlight as being an office-worker.

\section{B. Situated Role Assignment}

The three situations we consider here are depicted in the Fig. 3 below.

Fig. 3(a) depicts the outcome of the modeler's inquiry into the epistemic grounding of the image of a person boarding the train with a bag. Based on the semantics the service-designer assigns to this image will reveal the epistemic grounding of the categories bag, person and train. As explained in the figure, the semantics of this situation could well be inspired due to cultural influences, such as considering carrying personal belongings in a bag as part of a social norm enforcing civilized behavior. In that context, the categories do not seem to have any causal relevance to the phenomenon of mixed-mode of transport, unless we assume that conforming to social norms is also part of the design objective, which is a completely justified design constraint. Nevertheless, for the purpose of this illustration we discard that possibility. On the other hand, if we interpret the bike-in-the-bag imagery to convey the need to comply with local transport regulation, which requires bikes stored in the passenger area to stay in a bag at all times, then it does reveal the pragmatic basis of the imagery and, hence, assigns the categories a functional role. For example, in this case the person undertaking the role of the Bike User responsible for the outcomes arising out of the usage of the bike, as in storing the bag while on board the public transport. Another, pragmatic reasoning could be grounded in the person's concern for the inconvenience of the fellow passengers. In that case, the person may store the bike in the bag out his civic sense to avoid any injury to fellow commuters from the exposed sharp edges of the bike or the grease on the mechanical parts of the bike leaving stains on their clothes. Here the person can be modeled as undertaking the functional role of a Co-passenger.

Fig. 3(b) depicts the outcome of the modeler's inquiry into the epistemic grounding of the image of home to office commute. The three possible semantics explored for this situation are included here. First is a rationally oriented conceptualization, which is based on the logic that the categories of source and destination are always present whenever there is any reference to commute. Second is a pragmatic orientation, which reveals the issue related to the storing/parking of bike at home, thereby highlighting the role of the person as a Bike Owner concerned about the safety of her bike. Third is another pragmatic orientation, which reveals the issue of the person being able to avoid both the intra-city and inter-city traffic. In this case the semantics of the situation highlight the role of the person as a Biker and Passenger. It is important to note that some of the above pragmatically oriented interpretations of a situation required several refinements.

Fig. 3(c) depicts the outcome of the modeler's inquiry into the epistemic grounding of the image of the train as means of public transport. An initial interpretation of this situation could be grounded in empirical consideration, which is based purely on the observations that there is a 


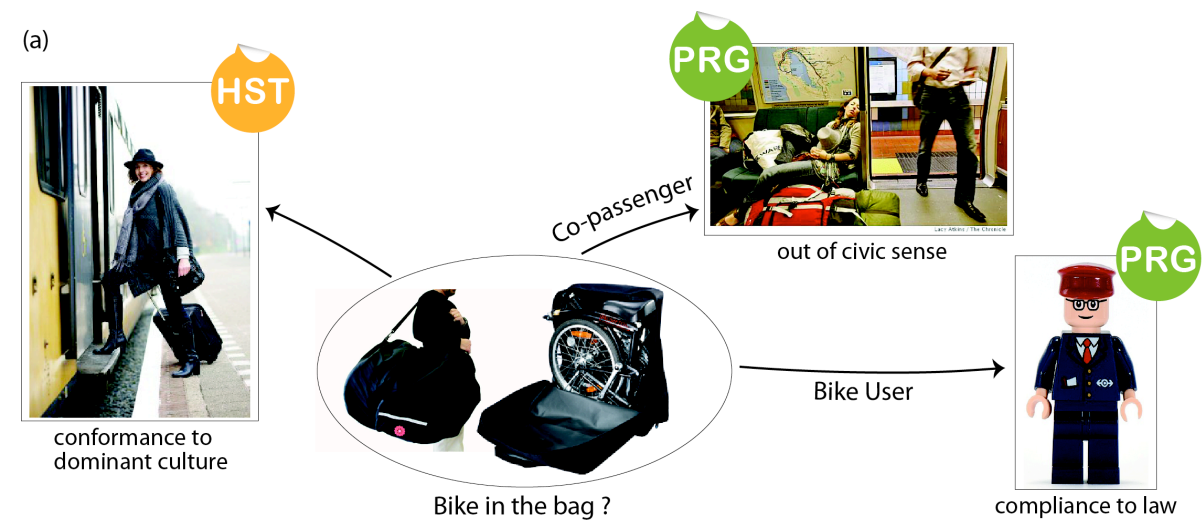

(b)
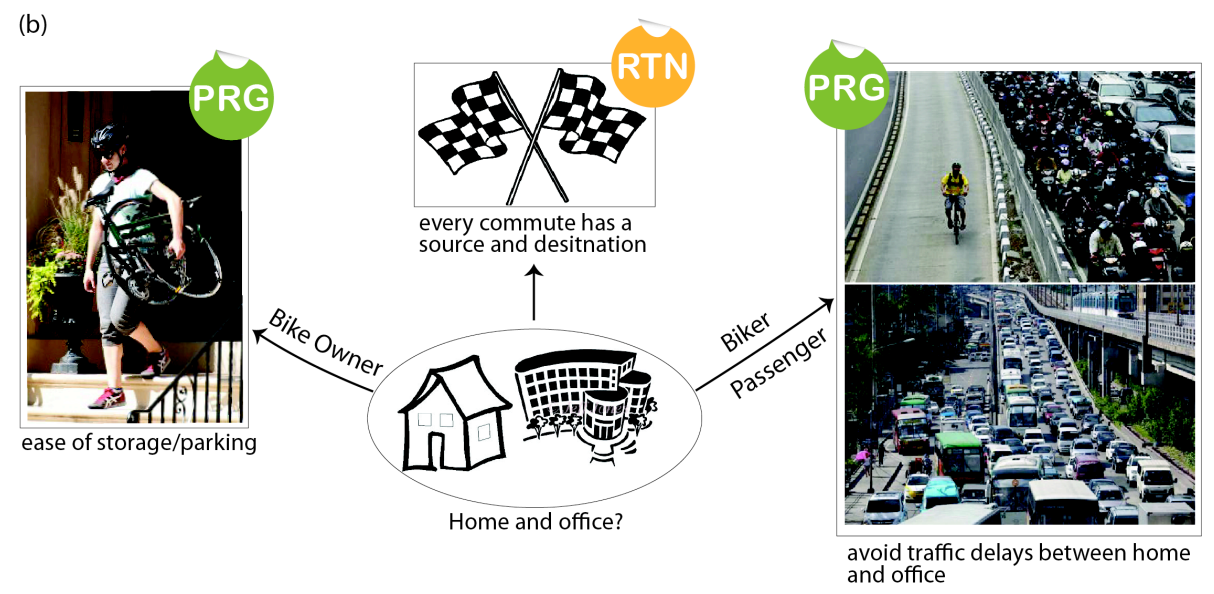

(c)
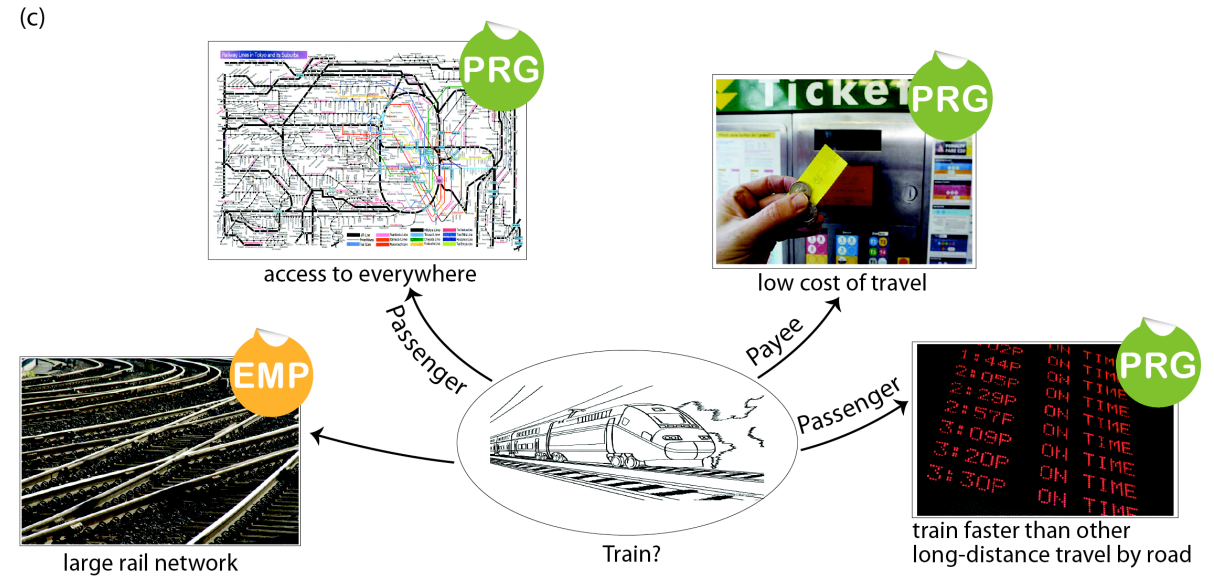

Historical grounding

Rational grounding

Empirical grounding

Pragmatic grounding

Figure 3: Critical reflection in action

large rail network in the area. Nevertheless, in the absence of any judgment it remains unclear how this rail network provides means of transport for people. It could be the case that the region has a large rail network to support freight movement, as is often the case in a port city, manufacturing hub, or an area rich in minerals. In an effort to identify the causal relevance of this observation, the service-designer might be inclined to explore other refinements of this situation. For example, further reflection may reveal the existence of a transport network that takes advantage of this large rail network to provide people access to everywhere in the sense of universal coverage. It is then easy to accept the causal relevance of train as a public-transport, which the user can take as part of the mixed-mode transport thereby assigning the functional role of Passenger to the user. This role is also discovered as part of critical reflection on the situation in 3(b). As it will be clear in the following subsection, we are using the role Passenger to refer to all the 


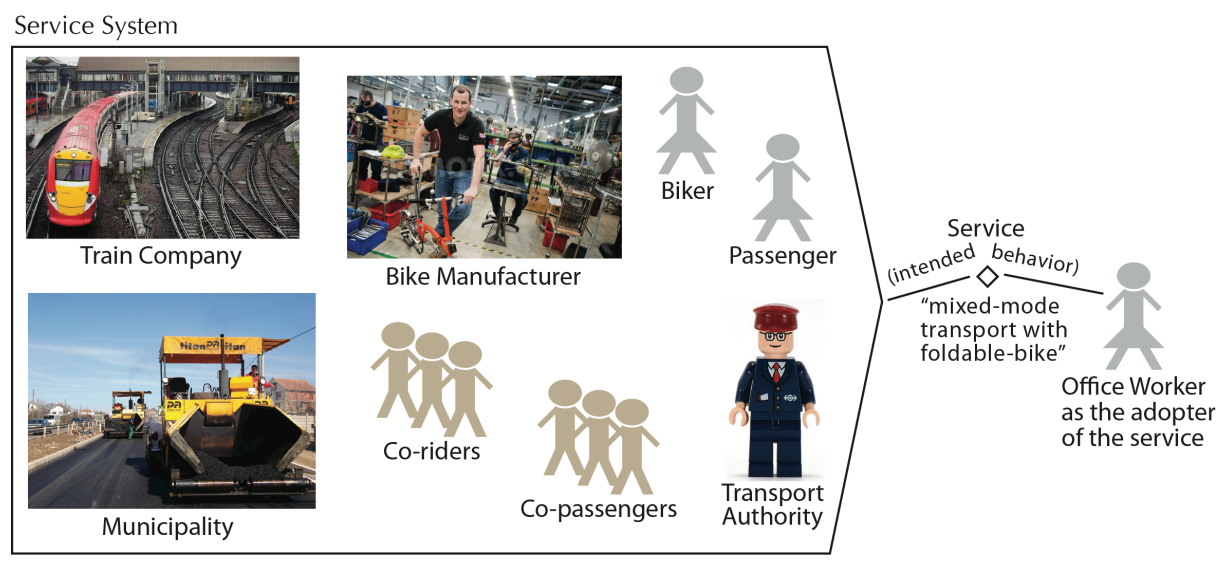

Figure 4: An example Service System as an outcome of situated modeling

cause-and-effect relations the user will participate to by virtue of her using the train as a means of public-transport. Fig. 3(c) includes another grounding - of the train being faster than other means of long-distance travel, which is considered to enforce the earlier role of user as a Passenger. The final refinement included here, with reference to the interpretation of the train as a means of public transport imagery, exposes its pragmatic grounding in the low cost of travel that the train may provide to commuters. In that case it highlights the user playing the functional role of the Payee of public-transport travel cost.

\section{Service System}

Based on the situations that were admitted during the informed imagination of the phenomenon of use of foldablebike as part of mixed-mode commute, including the three situations specifically discussed above, we present the service-system that specifies the behavior that the foldablebike manufacturer should realize to create value for the user in her role as an office-worker. Fig. 4 provides a visual depiction of this service-system. The Service-system specifies all the roles that the participating entities play in realizing the intended behavior. Thus, the inclusion of the Train Company, Bike Manufacturer and Municipality refers to all the functional roles that each of them undertakes as part of their participation to the phenomenon of mixed-mode commute using foldable-bike. Train Company here refers to the agency managing all aspects of the railways as a meansof-public transport, including rail network, rolling stock, commercial operations such as ticketing and legal compliance to relevant civil code. Bike Manufacturer refers to the company that designs, develops, produces and sells bike as a physical product. Municipality refers to the agency that is responsible for city infrastructure, including roads and parking places. In addition, we also highlighted two other roles undertaken by human actors other than the user. Co-rider refers to the group of people, excluding the user, on the road in their role of being part of the city commuter traffic. Similarly, Co-passenger refers to the group of people, excluding the user, in their role of being on board the same train as the user, or in general using the same rail transport network. As for the user, we represent her only in two roles - as a Biker and as a Passenger. These roles are higher-level roles, which together subsume all other functional roles that the user was shown to undertake in the three situations that we analyzed earlier.

\section{CONCLUSION}

A complete service specification requires answering the how, what and why of the service. How corresponds to the behavior that is to be realized as part of the service offering. What corresponds to the potential this behavior has to induce change. And why corresponds to the experience that is created by virtue of this change, thereby justifying why the service should be adopted. Identifying the servicesystem is the first step in this three-staged process of specifying the service.

\section{REFERENCES}

[1] M. A. Bedau, "Weak Emergence," Philosophical Perspectives: Mind, Causation, and World, vol. 11, pp. 375-399, 1997.

[2] L. W. Barsalou, "Situated simulation in the human conceptual system," Language and Cognitive Processes, vol. 18, pp. 513-562, 2003.

[3] B. Hjørland, "Concept theory," Journal of the American Society for Information Science and Technology, vol. 60, pp. 1519-1536, 2009.

[4] A. B. Saxena and A. Wegmann, "From Composites to Service Systems: The Role of Emergence in Service Design," presented at the IEEE International Conference on Systems, Man, and Cybernetics, Seoul, Korea, 2012.

[5] W. Croft and D. A. Cruse, Cognitive Linguistics. Cambridge, UK: Cambridge University Press, 2004.

[6] K. Koffa, Principles of Gestalt Psychology. London: Routledge \& Kegan Paul Ltd, 1935. 